\title{
Análisis del crecimiento de espinaca (Spinacia oleracea L.) bajo el efecto de diferentes fuentes y dosis de nitrógeno
}

\section{Growth analysis of spinach (Spinacia oleracea L.) plants under the effect of different sources and doses of nitrogen}

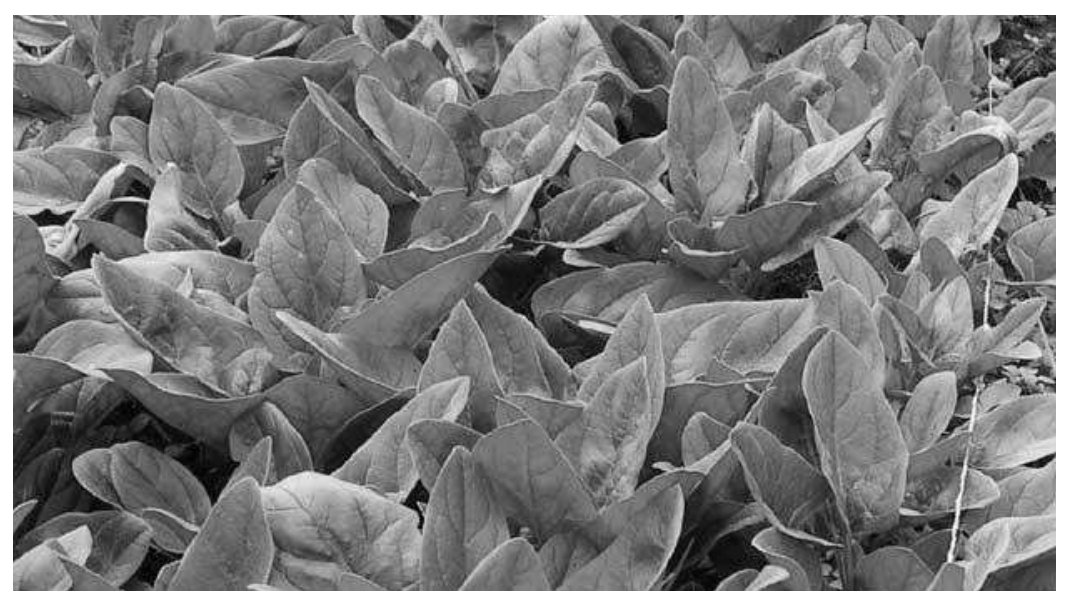

VERÓNICA HOYOS C. ${ }^{1,3}$

MARCELA RODRÍGUEZ2

JULIÁN FERNANDO CÁRDENAS-HERNÁNDEZ1

HELBER ENRIQUE BALAGUERA-LÓPEZ1

\section{RESUMEN}

Se estudió el comportamiento de variables fisiológicas e índices de crecimiento en espinaca bajo el efecto de diferentes dosis y fuentes de nitrógeno. Las plántulas de espinaca fueron trasplantadas a bolsas plásticas con suelo, las cuales crecieron bajo cubierta durante todo el experimento. Los tratamientos correspondieron a dos fuentes: urea y nitrato de amonio, y tres dosis de fertilización: 50, 100 y 150\%, de la recomendación del análisis de suelo. El nitrato de amonio fue la fuente con la cual se obtuvo mayor incremento en la salinidad del suelo. El nitrato de amonio a la dosis más alta generó la mayor área foliar, la mayor masa fresca total y el mayor índice de área foliar (IAF) a los 45 días después del trasplante (ddt). La masa seca de hojas y total de la planta presentaron los mayores valores con las dos fuentes en las dosis más altas. La tasa relativa de crecimiento (TRC), en general, fue decreciendo en el tiempo, donde nitrato de amonio al 50\% tuvo los valores más altos. La relación de área foliar (RAF) y el área foliar específico (AFE) disminuyeron progresivamente donde urea al $150 \%$ presentó la mayor disminución. La tasa de crecimiento de cultivo (TCC) tuvo un leve aumento en el tiempo, en la cual urea al 150\%, nitrato de amonio al 100\% y $150 \%$ presentaron mayores valores de esta tasa. La tasa de asimilación neta (TAN) disminuyó significativamente a través del tiempo, a los 7 ddt nitrato de amonio al 100\% obtuvo el mayor valor.

\footnotetext{
Programa de Maestría en Ciencias Agrarias con énfasis en Fisiología de Cultivos, Facultad de Agronomía, Universidad Nacional de Colombia, Bogotá (Colombia).

2 Programa de Maestría en Ciencias Agrarias con énfasis en Suelos y Aguas, Facultad de Agronomía, Universidad Nacional de Colombia, Bogotá (Colombia).

3 Autora para correspondencia.veronitahoyos@gmail.com
} 
Palabras clave adicionales: fertilización, curvas de crecimiento, tasas de crecimiento, conductividad eléctrica.

\section{ABSTRACT}

The behavior of physiological variables and growth rates in spinach under the effect of different doses and sources of nitrogen was studied. Spinach plantlets were transplanted into plastic bags filled with soil and located in a greenhouse, where remained during all the study. The treatments consisted of two fertilizer sources, urea and ammonium nitrate, and three doses, 50, 100 and $150 \%$, taking into account the recommendation of soil analysis. The treatments generated soil salinity, where ammonium nitrate was the source of the highest incidence. Ammonium nitrate at the highest dose was associated with the highest leaf area and total fresh mass. Dry mass of plants and leaf dry mass had the highest values with the two sources at the highest doses. Ammonium nitrate at the highest dose contributed to the highest leaf area, total fresh mass and leaf area index (LAI) at day 49 after transplanting (dat). Relative growth rate (RGR), in general, was decreasing in time, where ammonium nitrate at $50 \%$ showed the highest values. Leaf area ratio (LAR) and specific leaf area (SLA) progressively decreased, with urea at 150\% demonstrating the greatest decrease. The crop growth rate (CGR) had a slight increase in time, where $150 \%$ urea, ammonium nitrate at $100 \%$ and $150 \%$ had highest values of this rate. Net assimilation rate (NAR) decreased significantly over time and at 7 dat ammonium nitrate at $100 \%$ achieved the highest value.

Additional key words: fertilization, growth curves, growth rates, electrical conductivity.

Fecha de recepción: 21-10-2009

Aprobado para publicación: 30-11-2009

$\longrightarrow$

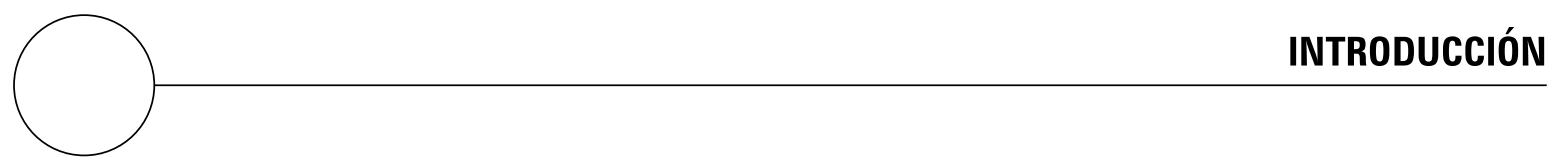

La espinaca (Spinacia oleracea L.) se distribuye principalmente en Europa, Asia y parte de América, el mayor productor mundial es China, con una participación del 90\%, le siguen Japón y Estados Unidos (Agrocadenas, 2006; Faostat, 2009). En Colombia los departamentos que producen esta hortaliza son Cundinamarca, Antioquia y Norte de Santander; a nivel nacional Cundinamarca participó con el $80 \%$ de la producción para el 2008, presentando un rendimiento de $16,7 \mathrm{t}$ ha- ${ }^{-1}$ en un área de 244 ha (Agronet, 2008).

La parte aérea de la planta es la comercializable a nivel mundial (Lucier et al., 2004; Agrocadenas, 2006). Sin embargo, en Colombia, la espinaca se comercializa en manojos, lo que incluye la raíz. Esta condición de venta genera que toda la planta se encuentre en óptimas condiciones tanto morfológicas, fisiológicas como sanitarias, con el fin de que cumplan con los requisitos establecidos en las normas de calidad para consumo. Para alcanzar estas condiciones se debe establecer un manejo agronómico adecuado durante todo el ciclo de cultivo, donde la nutrición de la planta es una práctica importante.

Una adecuada nutrición es la base esencial para la cantidad y calidad de los cultivos hortícolas, además, al comparar los requerimientos de estos cultivos su magnitud es superior a otros cultivos como cereales (Smatanová et al., 2004; Forero et al., 2008). La espinaca es una de las hortalizas más exigentes en nitrógeno, tanto para suplir sus necesidades bioquímicas, como para la obtención de una buena calidad, debido a que esta planta se comercializa principalmente en fresco, 
el nitrógeno cumpliría una función muy importante al aumentar la resistencia de las hojas a la manipulación (Suslow y Cantwell, 2002).

Los requerimientos nutricionales del cultivo de espinaca para la Sabana de Bogotá, fueron establecidos en el Centro de Investigaciones y Asesorías Agroindustriales de la Universidad de Bogotá Jorge Tadeo Lozano (CIAA-UJTL), por A. Forero (comunicación personal, 2009), estos son: $120 \mathrm{~kg} \mathrm{ha}^{-1} \mathrm{de}$ $\mathrm{N}, 45 \mathrm{~kg} \mathrm{ha}^{-1}$ de $\mathrm{P}_{2} \mathrm{O}_{5}, 200 \mathrm{~kg} \mathrm{ha}^{-1}$ de $\mathrm{K}_{2} \mathrm{O}, 116 \mathrm{~kg}$ $\mathrm{ha}^{-1}$ de $\mathrm{CaO}, 35 \mathrm{~kg} \mathrm{ha}^{-1}$ de $\mathrm{MgO}$ y $8 \mathrm{~kg} \mathrm{ha}^{-1}$ de S.

Adecuados contenidos de $\mathrm{N}$ incrementan las tasas de división y diferenciación celular y la actividad fotosintética, esto se traduce en mayor biomasa vegetativa o reproductiva en los cultivos por una alta eficiencia en la intercepción y conversión de la radiación (Uhart y Andrade, 1995). El contenido de $\mathrm{N}$ en la biomasa de las plantas varia desde $1 \%$ hasta $5 \%$ y dicho nutriente es absorbido bajo la forma de $\mathrm{NO}_{3}{ }^{-} \mathrm{O} \mathrm{NH}_{4}{ }^{+}$ (Marschner, 2002; Hammad et al., 2007). Es más frecuente que las plantas absorban el nitrógeno en forma de $\mathrm{NO}_{3}{ }^{-}$debido a que el $\mathrm{NH}_{4}{ }^{+}$es oxidado en $\mathrm{NO}_{3}{ }^{-}$con mucha rapidez por las bacterias nitrificantes (Salisbury y Ross, 2000). Sin embargo, fundamentando la absorción en el balance energético de la planta, el $\mathrm{NH}_{4}^{+}$sería la fuente preferida de $\mathrm{N}$ por el bajo costo energético para la síntesis de proteínas comparado con el $\mathrm{NO}_{3}^{-}$. Esto implica que las plantas que utilizan $\mathrm{NH}_{4}^{+}$ como fuente de $\mathrm{N}$ pueden tener mayores niveles de carbohidratos y proteínas respecto a las plantas que utilizan $\mathrm{NO}_{3}^{-}$. No obstante, no todas las especies se comportan de la misma manera cuando éstas dos fuentes de $\mathrm{N}$ se encuentran en el medio de cultivo (Echeverría y Sainz, 2005).

La salinidad de los suelos afecta el crecimiento de diversos cultivos (Marschner, 2002; Salisbury y Ross, 2000), entre ellos los cultivos hortícolas a nivel mundial (Carranza et al., 2009). Los efectos de la salinidad pueden manifestarse de diversas maneras, aunque existen tres aspectos importantes a resaltar como son: relaciones hídricas, donde la concentración de las sales solubles eleva la presión osmótica de la solución del suelo; el balance energético, al aumentar la presión osmótica de la solución, sufren una adaptación osmótica de sus células para poder absorber agua, llevando a cabo un mayor consumo de energía, produciendo un menor crecimiento en su altura; y nutrición, una elevada concentración de sales repercute sobre los niveles de absorción de algunos elementos nutritivos, principalmente porque disminuye la absorción de agua por las raíces, y por tanto de solutos, y por los fenómenos de antagonismo en la absorción y transporte de los iones (Marschner, 2002; Munns, 2002).

La conductividad eléctrica (CE) es la capacidad que tiene una sustancia de transmitir una corriente eléctrica (Bohn et al., 1993; Doerge et al., 2007). Para la agricultura esta medida corresponde a la cantidad de sales solubles tanto en el agua como en el suelo (Marschner, 1995; Nijensohn y Maffei, 1996; Oustan et al., 2007; Sposito, 2008), proporcionando un indicativo de la salinidad presente en el medio (Bohn et al., 1993; Sposito, 2008). Por las normas agrícolas un suelo con más de $4 \mathrm{dS} \mathrm{m}^{-1}$ de CE se considera como salino (Gartley, 1995).

El objetivo del presente trabajo fue realizar el análisis del crecimiento de plantas de espinaca bajo el efecto de diferentes dosis y fuentes de nitrógeno bajo condiciones de invernadero, con el fin de determinar la fuente y dosis de nitrógeno más adecuadas para el crecimiento de las plantas.

\section{MATERIALES Y MÉTODOS}

El experimento se desarrolló en los invernaderos de la Facultad de Agronomía de la Universidad Nacional de Colombia, Bogotá, ubicados a 2.556 msnm, temperatura promedio de $20^{\circ} \mathrm{C}$ y $80 \%$ de humedad relativa. Se utilizó un diseño experimental completamente al azar con seis tratamientos, los cuales se definieron teniendo en cuenta el análisis de suelos (tabla 1) y correspon- 
Tabla 1. Propiedades fisicoquímicas del suelo antes de la siembra.

\begin{tabular}{|c|c|c|c|c|c|c|c|c|c|c|c|c|}
\hline & $\mathrm{N}_{\mathrm{NO}}$ & $\mathrm{N}_{-} \mathrm{NH}_{4}$ & $\mathrm{~N}$-total & $\mathrm{Ca}$ & $\mathrm{K}$ & $\mathrm{Mg}$ & $\mathrm{Na}$ & $\mathrm{Al}$ & $\mathrm{CICe}$ & $\mathrm{P}$ & $\mathrm{S}$ & $\mathrm{CE}$ \\
\hline 5,3 & 4,1 & 35,8 & 39,9 & 6,46 & 1,93 & 2,29 & 0,21 & 0,08 & 11,0 & 61 & 23,9 & 0,79 \\
\hline
\end{tabular}

Fuente: Laboratorio de suelos CIAA-UJTL, Chía

dieron a dos fuentes fertilizantes de nitrógeno (urea y nitrato de amonio) y tres dosis de fertilización (recomendación análisis de suelos, 50\% recomendación y 150\% recomendación) (tabla 2), la cual se realizó según la metodología del ICA (1992). Cada tratamiento tuvo cuatro repeticiones, para un total de 24 unidades experimentales (UE), donde cada UE estuvo compuesta por 21 plantas. Se utilizaron plántulas de espinaca variedad Quinto de $25 \mathrm{~d}$ de germinadas, las cuales se trasplantaron en bolsas de polietileno con capacidad para $2 \mathrm{~L}$, como sustrato se utilizó suelo. La distancia de siembra fue $0,2 \times 0,2 \mathrm{~m}$.

Se realizaron siete muestreos, tomando tres plantas por UE en cada muestreo, y se determinaron las siguientes variables: número de hojas (medida directa); masa fresca de raíz y parte aérea (medición directa con balanza de precisión $0,01 \mathrm{~g}$ ); masa seca de raíz y parte aérea (medición directa con balanza de precisión $0,01 \mathrm{~g}$ después de someter las muestras a $75^{\circ} \mathrm{C}$ durante $48 \mathrm{~h}$ ); área foliar (medición directa mediante medidor de área foliar LI-3000; LI-COR Biosciences, Lincoln, NE). La conductividad eléctrica se midió al final del
Tabla 2. Descripción de los tratamientos evaluados.

\begin{tabular}{|l|r|r|}
\hline \multicolumn{1}{|c|}{ Fuente $^{1}$} & Dosis (g/planta) & Abreviatura \\
\hline Urea & 0,33 & U $50 \%$ \\
\hline Urea & 0,66 & U $100 \%$ \\
\hline Urea & 0,99 & U $150 \%$ \\
\hline Nitrato de amonio & 0,46 & N $50 \%$ \\
\hline Nitrato de amonio & 0,93 & N $100 \%$ \\
\hline Nitrato de amonio & 1,39 & N $150 \%$ \\
\hline
\end{tabular}

${ }^{1}$ Los restantes elementos se aplicaron según la recomendación.

ensayo mediante pasta de saturación y lectura con el conductímetro CG853 (Schott Instruments/SI Analytics GmbH, Mainz, Alemania). Además, se determinaron los parámetros de crecimiento siguiendo la metodología de Carranza et al. (2009) (tabla 3).

Se realizó un análisis de varianza (Anova) y se utilizó la prueba de comparación de medias de Tukey con una confiabilidad del 95\%, también se determinaron los modelos estadísticos de mayor ajuste para cada variable, para lo cual se utilizaron los programas Microsoft Excel 2003 y SAS v. 8.1e (Cary, NC.).

Tabla 3. Descripción de los parámetros de crecimiento que se midieron.

\begin{tabular}{|l|l|l|l|}
\hline \multicolumn{1}{|c|}{ Índice } & \multicolumn{1}{c|}{ Descripción } & \multicolumn{1}{c|}{ Fórmula } & \multicolumn{1}{c|}{ Unidades } \\
\hline TRC & Tasa relativa de crecimiento & $(1 / \mathrm{W})(\mathrm{dW} / \mathrm{dt})$ & $\mathrm{g} \mathrm{g}^{-1} \mathrm{~d}^{-1}$ \\
\hline IAF & Índice de área foliar & $\mathrm{L}_{\mathrm{A}} / \mathrm{P}$ & Adimensional \\
\hline TCC & Tasa de crecimiento del cultivo & $(1 / \mathrm{P})(\mathrm{dW} / \mathrm{dt})$ & $\mathrm{g} \mathrm{cm}^{-2} \mathrm{~d}^{-1}$ \\
\hline AFE & Área foliar específica & $\mathrm{L}_{\mathrm{A}} / \mathrm{LW}$ & $\mathrm{cm}^{2} \mathrm{~g}^{-1}$ \\
\hline RAF & Relación de área foliar & $\mathrm{L}_{\mathrm{A}} \mathrm{W}$ & $\mathrm{cm}^{2} \mathrm{~g}^{-1}$ \\
\hline TAN & Tasa de asimilación neta & $\left(1 / \mathrm{L}_{\mathrm{A}}\right)(\mathrm{dW} / \mathrm{dt})$ & $\mathrm{g} \mathrm{cm}^{-2} \mathrm{~d}^{-1}$ \\
\hline
\end{tabular}

$\mathrm{W}=$ masa seca total $(\mathrm{g}) ; \mathrm{LA}=$ área foliar $\left(\mathrm{cm}^{2}\right) ; \mathrm{P}=$ área de suelo $\left(\mathrm{cm}^{2}\right) ; \mathrm{LW}=$ masa seca foliar por planta $(\mathrm{g})$; $\mathrm{dW} / \mathrm{dt}=$ variación de la masa seca en función del tiempo. 


\section{RESULTADOS Y DISCUSIÓN}

\section{Conductividad eléctrica}

Durante el desarrollo del ensayo se observaron síntomas foliares típicos de estrés por salinidad, como desarrollo escaso de la planta, deformaciones y amarillamiento en las hojas. La aplicación de diferentes dosis de nitrógeno y fuentes de fertilización aumentaron la conductividad eléctrica del suelo, generando salinidad, siendo el tratamiento con nitrato de amonio a la mayor dosis el que con diferencias significativas presentó mayor valor de conductividad eléctrica (figura 1). La aplicación de fertilizantes puede afectar la salinidad de los suelos debido a que los iones acompañantes de algunos productos no son absorbidos en su totalidad, dejando residuos que elevan las sales del suelo, esta característica equivale al índice de salinidad $\left(\mathrm{NaNO}_{3}=100\right)$ que estos presenten. El nitrato de amonio presenta un índice de salinidad más alto que el de la urea $(104,7$ y 75,4 respectivamente), esto sumado al aumento en las dosis de fertilización incrementó la acu- mulación de sales en el suelo reflejado en un aumento en la CE (Guerrero, 2004). Igualmente la urea, por ser una forma orgánica no disociada en disolución, no aumenta la CE (Alarcón, 2008).

Los suelos son clasificados como salinos cuando tienen una conductividad mayor a $4 \mathrm{dS}^{-1}$ y su formación se debe generalmente a falta de drenaje y elevado porcentaje de evaporación, lo cual origina la mencionada acumulación de sales (Ibáñez, 2008). Al respecto, una de las causas de aumento de sales solubles en el suelo del presente ensayo fueron las altas temperaturas registradas en el invernadero (máxima $39^{\circ} \mathrm{C}$ ), ya que cuando existen sequías o altas temperaturas se producen altas evaporaciones, provocando la acumulación de sales en la solución del suelo (Chow et al., 1990).

\section{Análisis de crecimiento}

El crecimiento del cultivo de espinaca presentó una tendencia exponencial, reflejada por el comportamiento de las variables analizadas (tabla 4),

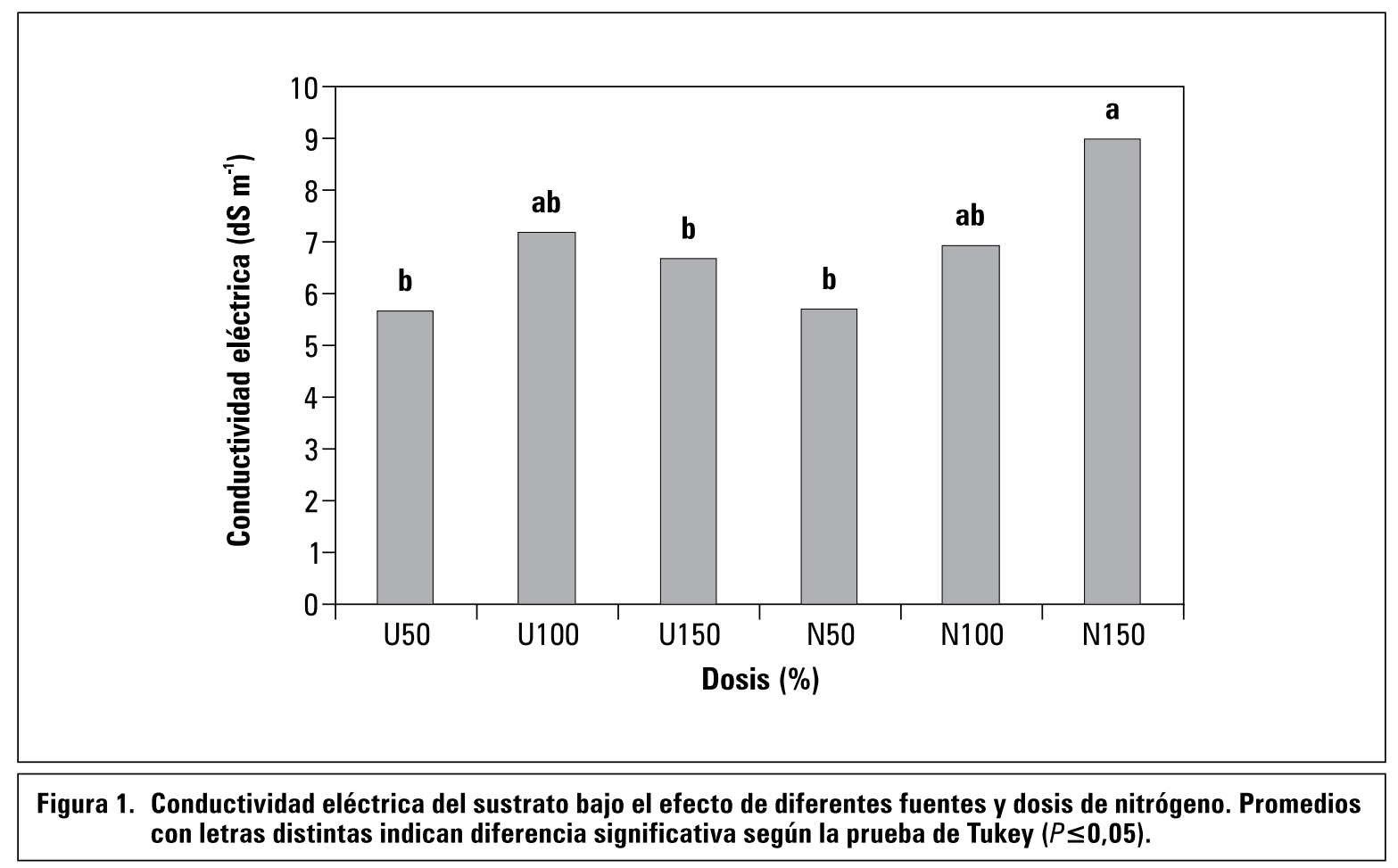


esto se da porque el cultivo no cumple la totalidad de ciclo de vida al momento de la comercialización, el cual correspondió al último muestreo realizado. El crecimiento de las especies vegetales presentan una curva sigmoidal, que presenta el tamaño acumulado en función del tiempo, la cual está compuesta por tres fases de crecimiento: logarítmico, lineal y de senescencia (Salisbury y Ross, 2000). Sin embargo, la espinaca alcanzó a presentar las dos primeras fases de crecimiento.

\section{Área foliar y número de hojas}

La variable área foliar presentó diferencias significativas a los 21 y $42 \mathrm{ddt}$, el tratamiento que generó mayor área foliar fue la fertilización con nitrato de amonio al $150 \%$. En la figura $2 \mathrm{~A}$ se observa el cambio de área foliar a través del tiempo para cada uno de los tratamientos, corroborando que el nitrato de amonio a la dosis más alta presenta mayor valor de esta variable durante el periodo de evaluación, siendo el valor a los 49 ddt de $91,64 \mathrm{~cm}^{2}$, mientras el tratamiento con urea al $50 \%$ para la misma fecha presentó el menor valor $\left(69,24 \mathrm{~cm}^{2}\right)$. Resultados similares se encontraron en lechuga donde a mayor dosis de nitrógeno hubo mayor desarrollo de área foliar (Grazia et al., 2001).

El tratamiento que presentó mayor área foliar (N 150\%) corresponde al tratamiento con mayor CE. Resultados opuestos se reportan en ensayos de cultivares de fresa, donde a mayor concentración de $\mathrm{NaCl}$ menor área foliar y menor número de hojas (Casierra-Posada y García, 2005), igualmente en plantas de feijoa a mayor concentración de salinidad en el suelos, éstas dos variables disminuyeron (Casierra-Posada y Rodríguez, 2006).

La tasa de crecimiento de las hojas depende de la continua e irreversible expansión de células jóvenes, las cuales son producidas por la división celular en los tejidos meristemáticos. De este modo, el suministro subóptimo de nutrientes podría afectar la tasa de crecimiento de las ho- jas por la inhibición de la tasa de producción y expansión de nuevas hojas (Neumann, 1997). La salinidad puede afectar principalmente la elongación foliar, y de ahí el desarrollo del área foliar fotosintética en algunas especies (Curtis y Läuchli, 1986) y la capacidad fotosintética en otras (Cramer et al., 1990). En el presente estudio, los tratamientos que presentaron la mayor área foliar (nitrato de amonio al 100\% y 150\%), fueron aquellos donde se presentó mayores valores de CE.

La variable "número de hojas" no presenta diferencias significativas durante el periodo de evaluación (figura 2B). Sin embargo, a los $49 \mathrm{ddt}$, el tratamiento de nitrato de amonio a 50\% mostró el mayor número de hojas y la fertilización con urea al 50\% el menor número de hojas, con los menores valores de área foliar para los dos tratamientos. Por otro lado, la fertilización con nitrato de amonio a dosis de $100 \%$ y $150 \%$ son las que presentan mayor valor de área foliar ( 89 y $91 \mathrm{~cm}^{2}$, respectivamente) y valores intermedios de número de hojas, indicando que bajo estos tratamientos las plantas de espinaca desarrollan menos hojas pero de mayor área (figura 2A).

\section{Masa seca y fresca}

La acumulación de masa seca y fresca de hojas y raíz, al igual que el área foliar presentaron un aumento exponencial en el tiempo (tabla 4), esto indica que la rapidez de crecimiento es baja al principio pero aumenta en forma continua (hasta $21 \mathrm{ddt}$; esta rapidez es proporcional al tamaño del organismo, cuanto mayor es éste, mayor será su crecimiento, correspondiente a la fase logarítmica de crecimiento. Posteriormente, la espinaca presentó un aumento en tamaño continuo a una velocidad constante y máxima (fase lineal) (Salisbury y Ross, 2000).

La variable masa fresca de hojas y raíz presentó diferencias significativas solo en dos fechas de evaluación, 14 y 21 ddt para hojas, y 14 y 42 ddt para raíz, dadas por la interacción fuente*dosis. Los tratamientos que reportan los valores más 
Tabla 4. Ecuaciones de regresión de los parámetros evaluados para determinar el crecimiento de plantas de espinaca bajo el efecto de diferentes dosis y fuentes de nitrógeno.

\begin{tabular}{|c|c|c|c|}
\hline Variable & Tratamientos & Modelo & $R^{2}$ \\
\hline \multirow{6}{*}{ Masa seca total } & Urea $50 \%$ & $y=0,0351 e^{0,0582 x}$ & 0,98 \\
\hline & Urea $100 \%$ & $y=0,0328 e^{0,0629 x}$ & 0,98 \\
\hline & Urea $150 \%$ & $y=0,0439 e^{0,0586 x}$ & 0,97 \\
\hline & Nitrato de amonio 50\% & $y=0,0442 e^{0,0548 x}$ & 0,97 \\
\hline & Nitrato de amonio $100 \%$ & $y=0,0434 e^{0,0595 x}$ & 0,98 \\
\hline & Nitrato de amonio $150 \%$ & $y=0,0434 e^{0,0595 x}$ & 0,98 \\
\hline \multirow{6}{*}{ Masa seca hojas } & Urea $50 \%$ & $y=0,0268 e^{0,0587 x}$ & 0,98 \\
\hline & Urea $100 \%$ & $y=0,0246 e^{0,064 x}$ & 0,98 \\
\hline & Urea $150 \%$ & $y=0,031 e^{0,0622 x}$ & 0,98 \\
\hline & Nitrato de amonio $50 \%$ & $y=0,0309 e^{0,0576 x}$ & 0,97 \\
\hline & Nitrato de amonio $100 \%$ & $y=0,0331 e^{0,0588 x}$ & 0,98 \\
\hline & Nitrato de amonio $150 \%$ & $y=0,0322 e^{0,0608 x}$ & 0,98 \\
\hline \multirow{6}{*}{ Masa seca raíz } & Urea $50 \%$ & $y=0,0268 e^{0,0587 x}$ & 0,98 \\
\hline & Urea 100\% & $y=0,0246 e^{0,064 x}$ & 0,98 \\
\hline & Urea $150 \%$ & $y=0,031 e^{0,0622 x}$ & 0,98 \\
\hline & Nitrato de amonio 50\% & $y=0,0309 e^{0,0576 x}$ & 0,97 \\
\hline & Nitrato de amonio $100 \%$ & $y=0,0331 e^{0,0588 x}$ & 0,98 \\
\hline & Nitrato de amonio $150 \%$ & $y=0,0322 e^{0,0608 x}$ & 0,98 \\
\hline \multirow{6}{*}{ Masa fresca total } & Urea $50 \%$ & $y=0,2894 e^{0,0578 x}$ & 0,96 \\
\hline & Urea 100\% & $y=0,2491 e^{0,0632 x}$ & 0,97 \\
\hline & Urea $150 \%$ & $y=0,394 e^{0,0529 x}$ & 0,93 \\
\hline & Nitrato de amonio $50 \%$ & $y=0,3946 e^{0,0512 x}$ & 0,91 \\
\hline & Nitrato de amonio $100 \%$ & $y=0,5536 e^{0,0445 x}$ & 0,87 \\
\hline & Nitrato de amonio $150 \%$ & $y=0,379 e^{0,0576 x}$ & 0,97 \\
\hline \multirow{6}{*}{ Área foliar } & Urea $50 \%$ & $y=5,874 e^{0,0529 x}$ & 0,94 \\
\hline & Urea 100\% & $y=5,8128 e^{0,0544 x}$ & 0,99 \\
\hline & Urea 150\% & $y=7,3006 e^{0,0502 x}$ & 0,99 \\
\hline & Nitrato de amonio 50\% & $y=6,8551 e^{0,0493 x}$ & 0,98 \\
\hline & Nitrato de amonio $100 \%$ & $y=7,6248 e^{0,051 x}$ & 0,98 \\
\hline & Nitrato de amonio $150 \%$ & $y=7,6248 e^{0,051 x}$ & 0,98 \\
\hline \multirow{6}{*}{ Número de hojas } & Urea $50 \%$ & $y=4,0256 e^{0,0269 x}$ & 0,96 \\
\hline & Urea $100 \%$ & $y=3,9715 e^{0,0274 x}$ & 0,94 \\
\hline & Urea $150 \%$ & $y=4,1906 e^{0,0271 x}$ & 0,93 \\
\hline & Nitrato de amonio 50\% & $y=4,4667 e^{0,0239 x}$ & 0,78 \\
\hline & Nitrato de amonio 100\% & $y=4,3154 e^{0,0262 x}$ & 0,89 \\
\hline & Nitrato de amonio $150 \%$ & $y=4,1382 e^{0,027 x}$ & 0,93 \\
\hline
\end{tabular}



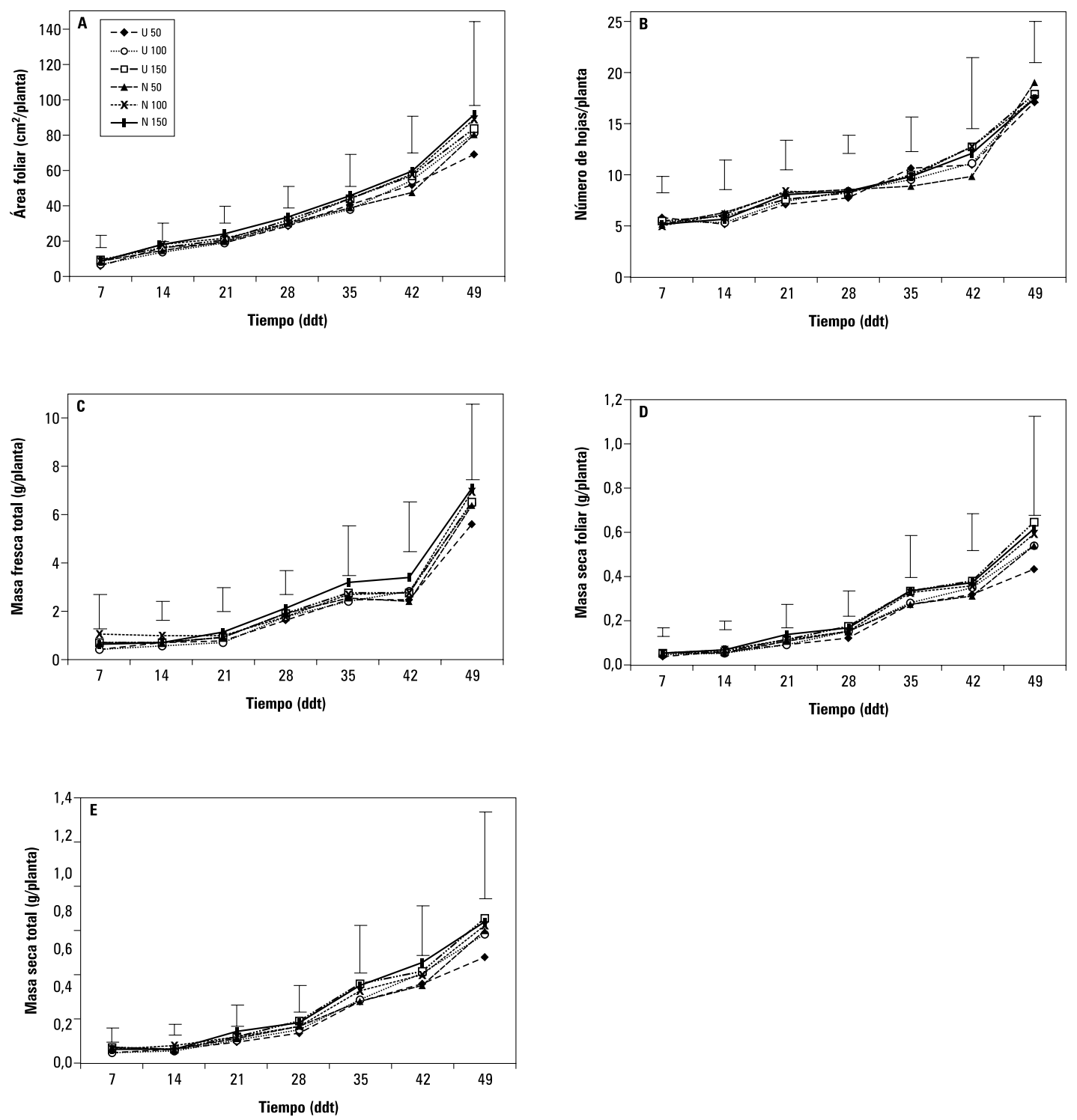

Figura 2. Comportamiento de A. Área foliar; B. Número de hojas; C. Masa fresca total; D. Masa seca foliar, y E. Masa seca total. La barra sobre los promedios representa el valor estadístico de la diferencia mínima significativa (LSD) para comparar los promedios, de acuerdo a la prueba de Tukey. Si las diferencias entre dos promedios son mayores al LSD, entonces habrá diferencia $(P \leq 0,05)$.

altos de masa fresca total son las dosis más altas de nitrato de amonio (100\% y $150 \%$; a los $49 \mathrm{ddt}$ valores de $6,92 \mathrm{~g}$ y $7,1 \mathrm{~g}$ respectivamente). Por el contrario, el tratamiento que presenta el menor valor fue urea a la menor dosis con 5,61 g (figura 2C). Grazia et al. (2001) reportan que plantas de lechuga presentaron menores valores de masa fresca y seca total cuando no hay aplicación de nitrógeno respecto a aquellas plantas que recibieron suministro de este elemento.

Para la variable masa seca de hojas los mejores tratamientos son las dos fuentes al 150\% (urea 150\% y nitrato de amonio 150\%; a los $49 \mathrm{ddt}$ valores de 0,65 g y 0,61 g respectivamente). Por el contrario, el tratamiento que presenta el menor valor 
fue urea a la menor dosis con 0,43 g (figura 2D).

La masa seca de hojas no presentó una respuesta directa a la salinidad debido a que los dos tratamientos con lo mayores valores no presentan $\mathrm{CE}$ similares (figura 1). Diferentes autores describen una disminución en el peso seco de las hojas causado por la salinidad, la cual genera una reducción de los parámetros relacionados con el crecimiento de las plantas (Robinson et al., 1983; Casierra-Posada y García, 2005). Sin embargo, estos cultivos se consideran como susceptibles a los altos niveles de conductividad eléctrica; por el contrario, la planta de espinaca se considera como la hortaliza que más tolera la salinidad (Burt, 2006).

La variable masa seca total no presentó diferencias significativas entre tratamientos. Los tratamientos que presentaron los mayores valores son urea $150 \%$ y nitrato de amonio $150 \%$ (a los 49 ddt valores de 0,77 g y 0,75 g respectivamente). Por el contrario el tratamiento que mostró el menor valor fue urea a la menor dosis con $0,43 \mathrm{~g}$ (figura 2E). Esta variable al igual que la masa seca de hojas no presentó una respuesta proporcional a la salinidad debido a que los dos tratamientos con los mayores valores no presentan CE similares (figura 1). Sin embargo, algunos autores reportan que las variables de crecimiento vegetativo tales como: masa seca, altura de la planta y área foliar, entre otras, son severamente afectadas por la presencia de sales (Alarcón et al., 1993), debido a que la salinidad altera y retrasa el crecimiento de las plantas ya que interviene en procesos fisiológicos como: fotosíntesis, conductancia estomática, ajuste osmótico, absorción de iones, síntesis de proteínas, síntesis de ácidos nucleicos, actividad enzimática y balance hormonal (Parés et al., 2008).

\section{Índice de área foliar}

Este índice tuvo un aumento continuo durante todo el periodo de evaluación, a los 49 ddt el tratamiento que presentó el mayor valor fue nitrato de amonio al $150 \%$, y los tratamientos con los valores menores fueron las dos fuentes en dosis del $50 \%$ (figura $3 \mathrm{~A}$ ). Esto indica que a mayores dosis de fertilización con nitrógeno mayor área foliar por unidad de suelo, Scheneiter (2000) afirma que la fertilización nitrogenada permite desarrollar mayores niveles de índice de área foliar y con ello capturar más luz en comparación con condiciones naturales, donde a mayor nivel de fertilización mayor el IAF en pastos. Igualmente, el IAF se vio afectado por efecto de la salinidad del suelo, resultados similares fueron encontrados por Carranza et al. (2009) en lechuga.

\section{Tasa relativa de crecimiento}

La TRC disminuyó durante el periodo de evaluación, donde los primeros muestreos presentaron los valores más altos, éste es el comportamiento típico de este índice, ya que a medida que crece la planta hay mayor acumulación de materia seca en relación a la producción de fotoasimilados. La TRC es la medida principal del análisis de crecimiento y se define como la ganancia de biomasa por unidad de biomasa y tiempo (Villar et al., 2004). La tendencia de este índice fue similar para todos los tratamientos, siendo la fertilización con urea al 100\% el más bajo y nitrato de amonio al 50\% el de mayor valor (figura 3B). Las altas dosis de fertilización favorecen la alta acumulación de materia seca comparadas con las bajas dosis (figura 2E).

En lechuga la tasa relativa de crecimiento disminuyó significativamente por el efecto de la radiación, independientemente del nivel de fertilización, sin registrarse diferencias significativas entre las dosis (Grazia et al., 2001). Debido a que las plantas de espinaca crecieron bajo condiciones de salinidad, muestran reducción en la TRC, datos similares fueron reportados por Carranza et al. (2009) donde la reducción es causada por las altas concentraciones de sales en el apoplasto de los tejidos afectando, directamente esta tasa de crecimiento. 


\section{Relación de área foliar}

Es un índice que relaciona la superficie foliar de la planta y la masa seca, se define como la fracción de masa seca total que corresponde a las hojas (Flórez et al., 2006; Carranza et al., 2009). La RAF disminuyó a medida que las plantas de espinaca fueron creciendo, debido a que la acumulación de materia seca es mayor comparada con el aumento del área foliar. La fertilización con urea al $150 \%$ presentó la mayor disminución, mientras la menor disminución se obtuvo con la misma fuente al 50\% (figura 3C). Los altos valores al inicio del desarrollo del cultivo se deben a que las plantas utilizan sus fotoasimilados en mayor proporción para el desarrollo y crecimiento de las áreas fotosintéticamente activas, generando gastos energéticos, lo que resulta en un menor peso (Carranza et al., 2009). La disminución de la RAF puede deberse a que al final del cultivo se presentaron hojas senescentes y con bordes necrosados por efecto de la salinidad, esto lleva a que haya menor área foliar para la realización de fotosíntesis pero continúan aumentando su masa seca.

\section{Área foliar específica}

Este índice presentó un compartamiento similar al RAF, disminuyendo a través del tiempo, donde urea al $150 \%$ tuvo una disminución más significativa y urea al 50\% una menor disminución con el valor más alto de AFE, y con la mayor densidad de hojas (figura 3D). El AFE es definida como la relación entre el área foliar y el peso de la hoja, es un índice de espesor y densidad de la hoja (Pérez et al., 2004). El área foliar específica es una de las principales variables que afectan el crecimiento de las plantas, ya que favorece cambios en la relación del área foliar y en la eficiencia fotosintética en el uso del nitrógeno (Bultynck et al., 1999).

Grazia et al. (2001) afirman que la nutrición nitrogenada afecta el desarrollo de la estructura foliar como consecuencia de su efecto sobre las variables de crecimiento (área foliar, duración del área foliar y tasa de expansión foliar) pero no modifica el área foliar por unidad de masa, es decir, el área foliar específica.

\section{Tasa de crecimiento del cultivo}

La fertilización con urea $150 \%$, nitrato de amonio $100 \%$ y $150 \%$ presentaron mayores valores de TCC (figura 3E), siendo estos los tratamientos más eficientes en la acumulación de masa seca por unidad de área de suelo a través del tiempo, ya que la TCC mide la ganancia de biomasa vegetal en el área de superficie ocupada por la planta (Hunt, 1990). Por otro lado el tratamiento que presentó el menor valor de la tasa fue urea a la dosis más baja (figura 3E), siendo esta la fertilización menos eficiente en la acumulación de masa seca. Carranza et al. (2009) en lechuga, obtuvieron un aumento progresivo de la TCC hasta los 35 ddt, y luego esta disminuyó hasta cosecha, dicha respuesta se asocia al efecto osmótico de la salinidad que contribuye a reducir la tasa de crecimiento.

\section{Tasa de asimilación neta}

La TAN disminuyó significativamente a través del tiempo y a medida que las plantas de espinaca fueron creciendo. El mayor valor se manifestó a los $7 \mathrm{ddt}$ para el tratamiento nitrato de amonio al $100 \%$ con un valor de $0,00025123 \mathrm{~g} \mathrm{~cm}^{-2} \mathrm{~d}^{-1}$ (figura 3F). A medida que la planta crece, existe sombreamiento por las hojas nuevas por sobreposición de estas, influyendo en la interceptación de la radiación fotosintéticamente activa, lo cual se ve reflejado en una disminución de la tasa de asimilación neta (Carranza et al., 2009), la cual expresa la eficiencia fotosintética. Se ha reportado que la salinidad reduce la tasa de crecimiento y en consecuencia la producción de los cultivos por una disminución de la eficiencia fotosintética (TAN), ya sea por disminución en la asimilación de fotosintatos, por reducción del conjunto de nucleótidos y el gasto adicional de 

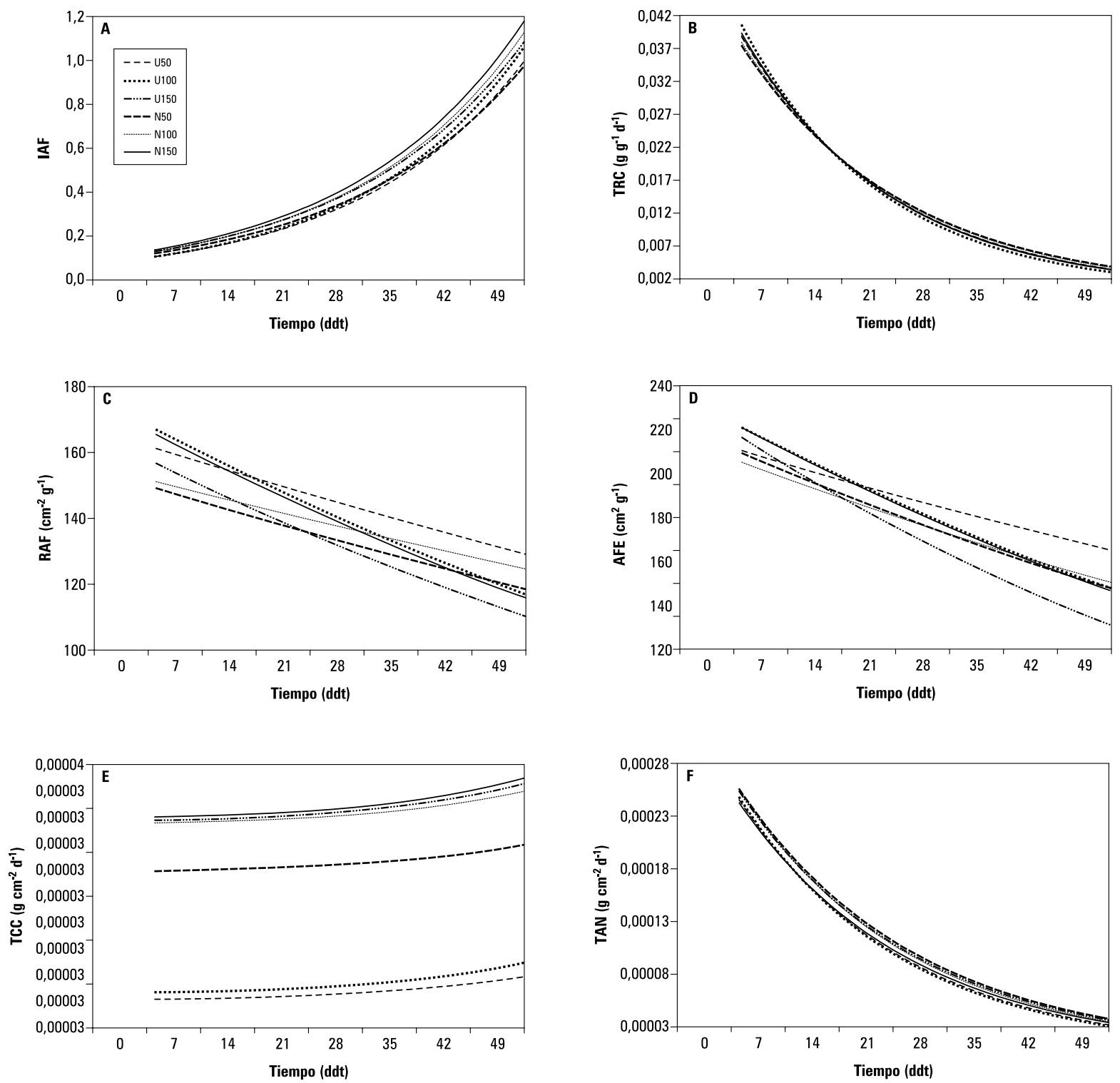

Figura 3. Parámetros de crecimiento en espinaca. A. Índice de área foliar (IAF); B. Tasa relativa de crecimiento (TRC); C. Relación de área foliar (RAF); D. Área foliar específica (AFE); E. Tasa de crecimiento del cultivo (TCC); F. Tasa de asimilación neta (TAN).

energía, por disminución de la conductancia estomática o por altos niveles de los iones sodio y cloro en el tejido foliar (Chartzoulakis y Klapaki, 2000). En especies de Phaseolus, el incremento de la salinidad disminuyó significativamente la TAN, al igual que la TCR, RAF y AFE (Bayuelo et al., 2008).

\section{CONCLUSIONES}

El crecimiento de las plantas de espinaca en el presente estudio se ajustó a un modelo exponencial. No se presentaron diferencias significativas en la utilización de las dos fuentes fertilizantes de nitrógeno y las tres dosis $50 \%$, 
$100 \%$ y $150 \%$ de la recomendación. Sin embargo, a mayor dosis de nitrógeno independiente de la fuente utilizada, se obtienen los mayores valores en masa seca de hojas y total. Las fuentes y dosis de nitrógeno tienen una incidencia en la conductividad eléctrica del suelo, donde el nitrato de amonio a la mayor dosis presenta mayor conductividad. La salinidad tiene mayor influencia en las variables área foliar y de hojas en el cultivo estudiado.

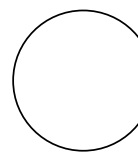

REFERENCIAS BIBLIOGRÁFICAS

Agrocadenas. 2006. Balance sectorial de Colombia. Observatorio de Competitividad Agrocadenas Colombia. Ministerio de Agricultura y Desarrollo Rural, Bogotá. En: http://agrocadenas.gov.co 6 pp.; consulta: octubre de 2009.

Agronet. 2008. Área y producción agrícola y pecuaria. Análisis - estadísticas. En: http://agronet.gov.co; consulta: octubre de 2009.

Alarcón, A.L. 2008. Abonado y salinidad en fertirrigación. En: http://infoagro.com; consulta: octubre de 2009.

Alarcón, J.; M. Sánchez-Blanco; M. Bolarin y A. Torrecillas. 1993. Water relations and osmotic adjustments in Lycopersicum esculentum and L. pennelli during shortterm salt exposure and recovery. Physiol. Plant. 89, 441-447.

Bayuelo J.S.; L. Bahena; L.M. Rodríguez y R. López. 2008. Crecimiento y respuestas fisiológicas de Phaseolus spp. en condiciones de salinidad. Revista Fitotecnia Mexicana 31(3), 213-223.

Bohn, H.; B. McNeal y G. O'Connor. 2001. Soil chemistry. 3rd ed. John Wiley and Sons, New York, NY.

Bultynck, L.; F. Fiorani y H. Lambers. 1999. Control of leaf growth and its role in determining variation in plant growth rate from an ecological perspective. Plant Biol. 1, 13-18.

Burt, J.R. 2006. Spinach in Australia. Development Officer. Tecnology transfer and Communications, South Perth.Department of Agriculture-WesternAustralia. En:http://agric.wa.gov.au; consulta:octubrede 2009.

Carranza, C.; O. Lanchero; D. Miranda y B. Chaves. 2009. Análisis del crecimiento de lechuga (Lactuca sativa L.) 'Batavia' cultivada en un suelo salino de la Sabana de Bogotá. Agron. Colomb. 27(1), 41-48.

Casierra-Posada, F. y N. García. 2005. Crecimiento y distribución de materia seca en cultivares de fresa (Fra- garia sp.) bajo estrés salino. Agron. Colomb. 23(1), 83-89.

Casierra-Posada, F. y S.Y. Rodríguez. 2006. Tolerancia de plantas de feijoa (Acca sellowiana [Berg] Burret) a la salinidad por NaCl. Agron. Colomb. 24(2), 258-265.

Chartzoulakis, K. y G. Klapaki. 2000. Response of two greenhouse pepper hybrids to $\mathrm{NaCl}$ salinity during different growth stages. Scientia Hort. 86(3), 247260.

Chow, W.S.; M.C. Ball y J.M. Anderson. 1990. Growth and photosynthetic responses of spinach to salinity: implications of $\mathrm{K}^{+}$nutrition for salt tolerance. Aust. J. Plant Physiol. 17, 563-578.

Cramer, G.R.; E. Epstein y A. Läuchli. 1990. Effects of sodium, potassium, and calcium on salt-stressed barley. I. Growth analysis. Physiol. Plant. 80, 83-88.

Curtis, P.S. y A. Läuchli. 1986. The role of leaf area development and photosynthetic capacity in determining growth of kenat under moderate salt stress. Aust. J. Plant Physiol. 18, 553-565.

Doerge, T.; N.R. Kitchen y E.D. Lund. 2007. Soil electrical conductivity mapping. Site-Specific Management Guidelines Series. En: International Plant Nutrition Institute. http://ppi-far.org/ssmg; consulta: septiembre de 2009.

Echeverría H.E. y H. Sainz. 2005. Nitrógeno en el suelo. pp. 69-97. En: Echevarría, H.E. y F. García (eds.). Fertilidad de suelos y fertilización de cultivos. Ediciones INTA, Buenos Aires.

Faostat. 2009. Series cronológicas y de datos con relación a la alimentación y agricultura. FAO, Roma.

Flórez, V.; D. Miranda; B. Chaves; L. Chaparro; C. Cárdenas y A. Farias. 2006. Parámetros considerados en el análisis de crecimiento en rosa y clavel en los sistemas de cultivo en suelo y en sustrato. pp. 43-51. En: 
Flórez, V.; A. de la C. Fernández; D. Miranda; B. Chaves y J.M. Guzmán (eds.). Avances sobre fertirriego en la floricultura colombiana. Facultad de Agronomía, Universidad Nacional de Colombia, Bogota.

Forero, A.; H. Escobary A. Medina. 2008. Caracterización de materiales orgánicos con aplicación potencial en la producción de hortalizas de hoja y brasicas en la Sabana de Bogotá p. 81. En: XIV Congreso Colombiano de la Ciencia del Suelo. Villavicencio, Colombia.

Gartley, K.L. 1995. Recommended soluble salts test. In: Soil testing the Northeastern United States. NEC67. http://ag.udel.edu; consulta: diciembre de 2009.

Grazia, J.; P.A. Tittonell y Á. Chiesa. 2001. Efecto de la época de siembra, radiación y nutrición nitrogenada sobre el patrón de crecimiento y el rendimiento del cultivo de lechuga (Lactuca sativa L.). Invest. Agr: Prod. Prot. Veg. 16(3), 355-365.

Guerrero, R. 2004. Propiedades generales de los fertilizantes. Manual técnico. 4a ed. Monomeros ColomboVenezolanos, Bogotá.

Hammad, S.A.; M.A. Abou-Seeda; A.M. El-Ghamry y E.M. Selim. 2007. Nitrate accumulation in spinach plants by using $\mathrm{N}$-fertilizer types in alluvial soil. J. Appl. Sci. Res. 3(7), 511-518.

Hunt, R. 1990. Basic growth analysis. Plant growth analysis for beginners. Unwin Hyman, Boston. 112 p.

Ibáñez, J.J. 2008. Tipos de suelos salinos. En: http://weblogs.madrimasd.org; consulta: octubre de 2009.

ICA. 1992. Fertilización de diversos cultivos. Quinta Aproximación. Manual de Asistencia Técnica No. 25. Bogotá.

Lucier, G.; J. Allshouse y B. Lin. 2004. Factors affecting spinach consumption in the United States. Electronic Outlook Report from the Economic Research Service. United States Department of Agriculture. En: http://ers.usda.gov 12 p.; consulta: octubre de 2009.

Marschner, H. 2002. Mineral nutrition of higher plants. Academic Press, London.

Munns, R. 2002. Comparative physiology of salt and water stress. Plant Cell Environ. 28, 239-250.

Neumann, P. 1997. Salinity resistance and plant growth revisited. Plant Cell Environ. 20, 1193-1198.

Nijensohn, L. y J.A. Maffei. 1996. Estimación de la salinidad y otras características edáficas a través de los volúmenes de sedimentación. Ciencia del Suelo 14, 119-121.
Oustan, S., A. Jafarzadeh y N. Aliasgharzad. 2007. Electrical conductivity as a salient factor in saline-sodic soils of Tabriz plain. Bioclimatology and Natural Hazards. International Scientific Conference, Pol'ana nad Detvou, Slovakia. http://cbks.cz; consulta: septiembre de 2009 .

Parés J.; M. Arizaleta; M.E. Sanabria y G. García. 2008. Efecto de los niveles de salinidad sobre la densidad estomática, índice estomático y el grosor foliar en plantas de Carica papaya L. Acta Bot. Venez. 31(1), 27-34.

Pérez, J.; E. García; J. Enríquez; A. Quero; J. Pérez y A. Garay. 2004. Análisis de crecimiento, área foliar específica y concentración de nitrógeno en hojas de pasto 'Mulato' (Brachiaria híbrido, cv.). Tec. Pecu. Mex. 42(3), 447-458.

Robinson, S.P.; W.S. Downtown y J.A. Millhouse. 1983. Photosynthesis and ion content of leaves and isolated chloroplasts of salt-stressed spinach. Plant Physiol. 73, 238-242.

Salisbury, F.B. y C.W. Ross. 2000. Fisiología vegetal. Grupo Editorial Iberoamérica, México.

Scheneiter, O. 2000. Las pasturas en suelos ganaderos. INTA, CRBAN, EEA Pergamino. Instituto Nacional de Tecnología Agropecuaria. En: http://inta.gob.ar; consulta: septiembre de 2009.

Smatanová, M.; R. Richtery J. Hlušek. 2004. Spinach and pepper response to nitrogen and sulphur fertilization. Plant Soil Environ. 50(7), 303-308.

Sposito, G. 2008. The chemistry of soils. $2^{\text {nd }}$ ed. Oxford University Press, Oxford, UK.

Suslow, T.R. y M. Cantwell. 2002. Spinach, recommendations for maintaining postharvest quality. Department of Vegetable Crops, University of California, Davis. En: http://postharvest.ucdavis.edu; consulta: octubre de 2009.

Uhart, S.A. y F.H. Andrade. 1995. Nitrogen deficiency and maize: I. Effects on crop growth, development, dry partitioning and kernel set. Crop Sci. 35, 1376 1383.

Villar, R.; J. Ruiz-Robleto; J.L. Quero; H. Poorter; F. Valladares y T. Maranon. 2004. Capítulo 7: Tasas de crecimiento en especies leñosas: aspectos funcionales e implicaciones ecológicas. pp. 193-226. En: Ecología del bosque mediterráneo en un mundo cambiante. Red Temática Globimet, Madrid. 